\title{
Cross-entropy measure on interval neutrosophic sets and its applications in Multicriteria decision making
}

Rıdvan Şahin

Bayburt University, Faculty of Education, Bayburt, 69000, Turkey

mat.ridone@gmail.com

Keywords:

Neutrosophic sets

Single valued neutrosophic sets

Interval neutrosophic sets

Cross-entropy

Multi criteria decision making

\begin{abstract}
The neutrosophic set model is an important tool for dealing with real scientific and engineering applications because it can handle not only incomplete information but also the inconsistent information and indeterminate information which exist commonly in real situations. In this paper, we firstly propose two practical methods to convert the interval neutrosophic sets into fuzzy sets and single valued neutrosophic sets, respectively. Then we define the interval neutrosophic cross-entropy in two different ways, which are based on extension of fuzzy crossentropy and the single valued neutrosophic cross-entropy. Additionally, a multicriteria decision-making method (MCDM) using the interval neutrosophic crossentropy measure between an alternative and the ideal alternative is developed in order to determine the order of the alternatives and choose most preferred one(s). Finally, an illustrative example is presented to verify the proposed approach and to demonstrate its effectiveness and practicality.
\end{abstract}

\section{Introduction}

Because of different types of uncertainties in real world, there are many of mathematics tools for handling incomplete, indeterminate and inconsistent information. Zadeh ([30]) proposed theory of fuzzy set which is applied successfully in various fields. Turksen ([21]) introduced the interval valued fuzzy set to overcome the difficulty to define the grade of membership of fuzzy set in some cases. Atanassov ([1]) proposed the concept of intuitionistic fuzzy set, which is a generalization of the concept of fuzzy set. Atanassov ([2],[3]) further generalized the intuitionistic fuzzy set theory and introduced the notion of interval-valued intuitionistic fuzzy set by combining the intuitionistic fuzzy set with interval valued fuzzy set, which is characterized by a membership function and a non-membership function whose values are intervals rather than real numbers. Then Smarandache ([18],[19]) defined the neutrosophic sets which are more general than the aforementioned sets. In a neutrosophic set, each element of the universe has a degree of truth, indeterminacy and falsity respectively. The indeterminacy value of neutrosophic sets is independent of truth and falsity values while the indeterminacy or hesitation margin is dependent of the degree of belongingness and degree of non-belongingness in the case intuitionistic fuzzy set. Neutrosophic sets have been successful used in image processing field, such as image thresholding and image segmentation ([7], [9]). But, a neutrosophic set will be difficult to apply in real scientific and engineering fields. Therefore, Wang et al. ([24], [25]) proposed the concepts of single valued neutrosophic set and interval neutrosophic set, which are an instance of a neutrosophic set, and provided them set-theoretic operators and various properties. Then, single valued neutrosophic sets present uncertainty, imprecise, inconsistent and incomplete information existing in real world. Also, it would be more suitable to handle indeterminate information and inconsistent information. Recently, some researchers have shown great interest in the single valued neutrosophic set theory and applied it to the field of decision making. Ye ([26]) proposed correlation coefficients between single valued neutrosophic sets and applied them to multiple attribute decision-making problems with single valued neutrosophic information. Furthermore, Ye ([29]) introduced the concept of a simplified 
neutrosophic set, which is a subclass of a neutrosophic set and includes the concepts of interval neutrosophic set and single valued neutrosophic set, and defined some operational laws of simplified neutrosophic sets, and then he proposed simplified neutrosophic weighted averaging operator and simplified neutrosophic weighted geometric operator and applied them to multi-criteria decision-making problems under the simplified neutrosophic environment. Peng et al. ([13]) point out that some operations defined by Ye ([29]) may also be invalid. They defined the novel operations and aggregation operators and applied them to multi criteria decision making problems. Liu and Wang ([10]) defined a single-valued neutrosophic normalized weighted Bonferroni mean operator. Şahin and Küçük ([17]) proposed the concept of neutrosophic subsethood based on distance measure for single valued neutrosophic sets. Recently, Peng et al. ([14]) defined the multi-valued neutrosophic sets and proposed two aggregation operators such that weighted average operator and the weighted geometric operator for solving a multi criteria decision making problem.

On the other hand, since the degree of truth, falsity and indeterminacy about a certain statement can not be defined exactly in the real situations, Wang et al. ([25]) generalized the concept of single valued neutrosophic set to the interval neutrosophic set which is characterized by the degree of truth, falsity and indeterminacy whose values are intervals rather than real numbers. After the pioneering work of Wang et al. ([25]), the interval neutrosophic set has received much attention in the literature. Ye ([27]) introduced the Hamming and Euclidean distances between interval neutrosophic sets and the distances-based similarity measures and applied them to multiple attribute decision-making problems with interval neutrosophic information. Broumi and Smarandache ([4]) proposed correlation coefficients between interval valued neutrosophic sets. Liu and shi ([11]) combined the interval neutrosophic sets and interval-valued hesitant fuzzy sets, and propose the concept of the interval neutrosophic hesitant sets. Also they developed some new aggregation operators for the interval neutrosophic hesitant fuzzy information, including interval neutrosophic hesitant fuzzy generalized weighted operator, interval neutrosophic hesitant fuzzy generalized ordered weighted operator, and interval neutrosophic hesitant fuzzy generalized hybrid weighted operator, and discussed some properties. Zhang et al. ([32]) introduced two interval neutrosophic aggregation operators and applied them to multicriteria decision-making problems with interval neutrosophic information.

Entropy, as a very important notion for measuring fuzziness degree or uncertain information in fuzzy set theory, has received great attention in the past decades. In 1968 ([31]), Zadeh first introduced the entropy of fuzzy event. Later, Deluca and Termini ([8]) presented some axioms to describe the fuzziness degree of fuzzy set, with which a fuzzy entropy based on Shannon's function ([16]) was proposed. After that, many other researchers have studied the fuzzy entropy in different ways. Bhandari and Pal ([6]) made a survey on information measures on fuzzy sets and gave some new measures of fuzzy entropy. Burillo and Bustince ([5]) presented an entropy on interval-valued fuzzy sets and intuitionistic fuzzy sets. Szmidt and Kacprzyk ([20]) proposed an entropy measure for intuitionistic fuzzy set by employing a geometric interpretation of intuitionistic fuzzy sets. Wei et al. ([23]) discussed an entropy measure for interval-valued intuitionistic fuzzy sets, which generalizes entropy measures defined for intuitionistic fuzzy sets. Majumdar et al. ([12]) used the entropy to solve a multi criteria decision making problem under single valued neutrosophic environment. Cross-entropy is used to measure the discrimination information between objects, according to Shannon's inequality ([16]). Shang and Jiang ([15]) defined the fuzzy cross-entropy between two fuzzy sets. Vlachos and Sergiadis ([22]) gave a definition of intuitionsitic fuzzy cross entropy and proved a mathematical connection between the notions of entropy for fuzzy sets and intuitionistic fuzzy sets in terms of fuzziness and intuitionism. For interval-valued fuzzy set, Zhang ([33]) defined the concepts of entropy and cross-entropy for interval valued intuitionistic sets and discussed its some properties. Ye ([28]) proposed the cross-entropy on single valued neutrosophic sets and defined the concept of single valued neutrosophic cross-entropy. 
Although some distance measures, similarity measures and correlation measures of interval neutrosophic sets have been presented in ([4], [12], [27], [32]) recently, there are no investigation on the entropy and crossentropy measures of interval neutrosophic sets. As we know, characterization of uncertainty for interval neutrosophic sets are important issues that affect the management of uncertainty information in most realworld system models involving imperfect information with interval neutrosophic set values. Thus, it focuses us to study an useful cross-entropy measure of interval neutrosophic set for the possible applications in many real-life areas such as pattern recognition, cluster analysis, image segment. In this paper, we firstly propose two practical methods to convert the interval neutrosophic sets into fuzzy sets and single valued neutrosophic sets. In addition, a cross-entropy of interval neutrosophic set is presented to determine the information measure for discrimination between two interval neutrosophic sets. Finally, an application of the proposed cross-entropy of interval neutrosophic sets is established in a decision making problem.

\section{Preliminaries}

In the following we give a brief review of some preliminaries.

\subsection{Neutrosophic set}

Definition 2.1 ([19]) Let $X$ be a space of points (objects) and $x \in X$. A neutrosophic set $A$ in $X$ is defined by a truth-membership function $T_{A}(x)$, an indeterminacy-membership function $I_{A}(x)$ and a falsity-membership function $F_{A}(x) . T_{A}(x), I_{A}(x)$ and $F_{A}(x)$ are real standard or real nonstandard subsets of $] 0^{-}, 1^{+}$[. That is $\left.T_{A}(x): X \rightarrow\right] 0^{-}, 1^{+}\left[, I_{A}(x): X \rightarrow\right] 0^{-}, 1^{+}\left[\right.$and $\left.F_{A}(x): X \rightarrow\right] 0^{-}, 1^{+}[$. There is not restriction on the sum of $T_{A}(x), I_{A}(x)$ and $F_{A}(x)$, so $0^{-} \leq \sup T_{A}(x) \leq \sup I_{A}(x) \leq \sup F_{A}(x) \leq 3^{+}$.

Definition 2.2 ([19]) The complement of a neutrosophic set $A$ is denoted by $A^{c}$ and is defined as $T_{A}^{c}(x)=$ $\left\{1^{+}\right\} \ominus T_{A}(x), I_{A}^{c}(x)=\left\{1^{+}\right\} \ominus I_{A}(x)$ and $F_{A}^{c}(x)=\left\{1^{+}\right\} \ominus F_{A}(x)$ for all $x \in X$.

Definition 2.3 ([19]) A neutrosophic set $A$ is contained in the other neutrosophic set $B, A \subseteq B$ iff $\inf T_{A}(x) \leq$ $\inf T_{B}(x), \sup T_{A}(x) \leq \sup T_{B}(x), \inf I_{A}(x) \geq \inf I_{B}(x), \sup I_{A}(x) \geq \sup I_{B}(x)$ and $\inf F_{A}(x) \geq \inf F_{B}(x)$, $\sup F_{A}(x) \geq \sup F_{B}(x)$ for all $x \in X$.

In the following, we will adopt the representations $u_{A}(x), p_{A}(x)$ and $v_{A}(x)$ instead of $T_{A}(x), I_{A}(x)$ and $F_{A}(x)$, respectively.

\subsection{Single valued neutrosophic sets}

A single valued neutrosophic set has been defined in ([24]) as follows:

Definition 2.4 ([24]) Let $X$ be a universe of discourse. A single valued neutrosophic set $A$ over $X$ is an object having the form

$$
A=\left\{\left\langle x, u_{A}(x), p_{A}(x), v_{A}(x)\right\rangle: x \in X\right\},
$$

where $u_{A}(x): X \rightarrow[0,1], p_{A}(x): X \rightarrow[0,1]$ and $v_{A}(x): X \rightarrow[0,1]$ with $0 \leq u_{A}(x)+p_{A}(x)+v_{A}(x) \leq 3$ for all $x \in X$. The values $u_{A}(x), p_{A}(x)$ and $v_{A}(x)$ denote the truth- membership degree, the indeterminacymembership degree and the falsity membership degree of $x$ to $A$, respectively.

Definition 2.5 ([24]) The complement of a single valued neutrosophic set $A$ is denoted by $A^{c}$ and is defined as $u_{A}^{c}(x)=v(x), p_{A}^{c}(x)=1-p_{A}(x)$, and $v_{A}^{c}(x)=u(x)$ for all $x \in X$. That is,

$$
A^{c}=\left\{\left\langle x, v_{A}(x), 1-p_{A}(x), u_{A}(x)\right\rangle: x \in X\right\} .
$$

Definition 2.6 ([24]) A single valued neutrosophic set $A$ is contained in the other single valued neutrosophic set $B, A \subseteq B$, iff $u_{A}(x) \leq u_{B}(x), p_{A}(x) \geq p_{B}(x)$ and $v_{A}(x) \geq v_{B}(x)$ for all $x \in X$. 
Definition 2.7 ([24]) Two single valued neutrosophic sets $A$ and $B$ are equal, written as $A=B$, iff $A \subseteq B$ and $B \subseteq A$. Moreover, we denote the family of all the single valued neutrosophic sets by $S V N S(X)$.

\subsection{Interval neutrosophic sets}

An interval neutrosophic set is a model of a neutrosophic set, which can be used to handle uncertainty in fieds of scientific, environment and engineering. We introduce the definition of an interval neutrosophic set as follows.

Definition 2.7 ([25]) Let $X$ be a space of points (objects) and Int[0,1] be the set of all closed subsets of $[0,1]$. An interval neutrosophic $A$ in $X$ is defined with the form

$$
A=\left\{\left\langle x, u_{A}(x), p_{A}(x), v_{A}(x)\right\rangle: x \in X\right\}
$$

where $u_{A}(x): X \rightarrow \operatorname{int}[0,1], \quad p_{A}(x): X \rightarrow \operatorname{int}[0,1]$ and $v_{A}(x): X \rightarrow \operatorname{int}[0,1] \quad$ with $0 \leq \sup u_{A}(x)+$ $\sup p_{A}(x)+\sup v_{A}(x) \leq 3$ for all $x \in X$. The intervals $u_{A}(x), p_{A}(x)$ and $v_{A}(x)$ denote the truthmembership degree, the indeterminacy-membership degree and the falsity membership degree of $x$ to $A$, respectively.

For convenience, if let $u_{A}(x)=\left[u_{A}^{L}(x), u_{A}^{U}(x)\right], p_{A}(x)=\left[p_{A}^{L}(x), p_{A}^{U}(x)\right]$ and $v_{A}(x)=\left[v_{A}^{L}(x), v_{A}^{U}(x)\right]$, then

$$
A=\left\{\left\langle x,\left[u_{A}^{L}(x), u_{A}^{U}(x)\right],\left[p_{A}^{L}(x), p_{A}^{U}(x)\right],\left[v_{A}^{L}(x), v_{A}^{U}(x)\right]\right\rangle: x \in X\right\}
$$

with the condition, $0 \leq \sup u_{A}^{U}(x)+\sup p_{A}^{U}(x)+\sup v_{A}^{U}(x) \leq 3$ for all $x \in X$. Here, we only take the subunitary interval of $[0,1]$. Therefore, an interval neutrosophic set is clearly neutrosophic set.

Definition 2.8 ([25]) Let INS(X) denote the family of all the interval neutrosophic sets in universe $X$, assume $A, B \in I N S(X)$ such that

$$
\begin{aligned}
& A=\left\{\left\langle x,\left[u_{A}^{L}(x), u_{A}^{U}(x)\right],\left[p_{A}^{L}(x), p_{A}^{U}(x)\right],\left[v_{A}^{L}(x), v_{A}^{U}(x)\right]\right\rangle: x \in X\right\} \\
& B=\left\{\left\langle x,\left[u_{B}^{L}(x), u_{B}^{U}(x)\right],\left[p_{B}^{L}(x), p_{B}^{U}(x)\right],\left[v_{B}^{L}(x), v_{B}^{U}(x)\right]\right\rangle: x \in X\right\}
\end{aligned}
$$

then some operations can be defined as follows:

(1) $A \cup B=\left\{\left\langle x,\left[\max \left\{u_{A}^{L}(x), u_{B}^{L}(x)\right\}, \max \left\{u_{A}^{U}(x), u_{B}^{U}(x)\right\}\right]\right.\right.$,

$$
\left.\left.\left[\min \left\{p_{A}^{L}(x), p_{B}^{L}(x)\right\}, \min \left\{p_{A}^{U}(x), p_{B}^{U}(x)\right\}\right],\left[\min \left\{v_{A}^{L}(x), v_{B}^{L}(x)\right\}, \min \left\{v_{A}^{U}(x), v_{B}^{U}(x)\right\}\right]\right\rangle: x \in X\right\} ;
$$

(2) $A \cap B=\left\{\left\langle x,\left[\min \left\{u_{A}^{L}(x), u_{B}^{L}(x)\right\}, \min \left\{u_{A}^{U}(x), u_{B}^{U}(x)\right\}\right]\right.\right.$,

$$
\left.\left.\left[\max \left\{p_{A}^{L}(x), p_{B}^{L}(x)\right\}, \max \left\{p_{A}^{U}(x), p_{B}^{U}(x)\right\}\right],\left[\max \left\{v_{A}^{L}(x), v_{B}^{L}(x)\right\}, \max \left\{v_{A}^{U}(x), v_{B}^{U}(x)\right\}\right]\right\rangle: x \in X\right\} ;
$$

(3) $A^{c}=\left\{\left\langle x,\left[v_{A}^{L}(x), v_{A}^{U}(x)\right],\left[1-p_{A}^{U}(x), 1-p_{A}^{L}(x)\right],\left[u_{A}^{L}(x), u_{A}^{U}(x)\right]\right\rangle: x \in X\right\}$;

(4) $A \subseteq B$, iff $u_{A}^{L}(x) \leq u_{B}^{L}(x), u_{A}^{U}(x) \leq u_{B}^{U}(x), p_{A}^{L}(x) \geq p_{B}^{L}(x), p_{A}^{U}(x) \geq p_{B}^{U}(x)$ and $v_{A}^{L}(x) \geq v_{B}^{L}(x)$, $v_{A}^{U}(x) \geq v_{B}^{U}(x)$ for all $x \in X$.

(5) $A=B$, iff $A \subseteq B$ and $B \subseteq A$.

Now, we define the cross-entropy measure of interval neutrosophic sets.

\section{Cross-entropy for interval neutrosophic sets}

In this section we present two different approaches to calculate the cross-entropy of interval neutrosophic sets, which are based on the fuzzy cross-entropy and interval neutrosophic cross entropy. We first introduce the concepts of cross-entropy and discrimination information measures between two fuzzy sets which were proposed by Shang and Jiang ([15]), and Bhandari and Pal ([6]), respectively. 
Definition 3.1 ([6]) Let $A$ and $B$ are two fuzzy sets in universe $X=\left\{x_{1}, x_{2}, \ldots x_{n}\right\}$. The fuzzy information for discrimination of $A$ from $B$ is defined as follows:

$$
K_{F S}(A, B)=\sum_{i=1}^{n}\left(\mu_{A}\left(x_{i}\right) \ln \frac{\mu_{A}\left(x_{i}\right)}{\mu_{B}\left(x_{i}\right)}+\left(1-\mu_{A}\left(x_{i}\right)\right) \ln \frac{1-\mu_{A}\left(x_{i}\right)}{1-\mu_{B}\left(x_{i}\right)}\right) .
$$

To overcome some drawbacks in formula (1), Shang and jiang ([15]) proposed a modified version of it called as fuzzy cross entropy.

Definition 3.3 Assume that $A=\left\langle\mu_{A}\left(x_{1}\right), \mu_{A}\left(x_{2}\right), \ldots, \mu_{A}\left(x_{n}\right)\right\rangle$ and $B=\left\langle\mu_{B}\left(x_{1}\right), \mu_{B}\left(x_{2}\right), \ldots, \mu_{B}\left(x_{n}\right)\right\rangle$ are two fuzzy sets in the universe of discourse $X=\left\{x_{1}, x_{2}, \ldots, x_{n}\right\}$. The fuzzy cross entropy of $A$ from $B$ is defined as follows:

$$
H_{F S}(A, B)=\sum_{i=1}^{n}\left(\mu_{A}\left(x_{i}\right) \log _{2} \frac{\mu_{A}\left(x_{i}\right)}{\frac{1}{2}\left(\mu_{A}\left(x_{i}\right)+\mu_{B}\left(x_{i}\right)\right)}+\left(1-\mu_{A}\left(x_{i}\right)\right) \log _{2} \frac{1-\mu_{A}\left(x_{i}\right)}{1-\frac{1}{2}\left(\mu_{A}\left(x_{i}\right)+\mu_{B}\left(x_{i}\right)\right)}\right)
$$

which indicates the degree of discrimination of $A$ from $B$.

However, $H_{F S}(A, B)$ is does not have symmetric property with respect to its arguments. Shang and Jiang ([15]) defined a symmetric discrimination information measure as $I_{F S}(A, B)=H_{F S}(A, B)+H_{F S}(B, A)$. Moreover, there are $I_{F S}(A, B) \geq 0$ and $I(A, B)=0$ iff $A=B$.

The information carried by the truth-membership, the indeterminacy-membership, and the falsitymembership in single valued neutrosophic sets A and B can be considered as fuzzy spaces with the three elements. Recently, Ye ([28]) extended the cross-entropy and symmetric discrimination information measures between two fuzzy sets to single valued neutrosophic sets and defined the concept of single valued neutrosophic cross entropy, which is a generalization of fuzzy cross entropy as follows:

Definition 3.4 Assume that $A=\left\langle u_{A}\left(x_{i}\right), p_{A}\left(x_{i}\right), v_{A}\left(x_{i}\right)\right\rangle$ and $B=\left\langle u_{B}\left(x_{i}\right), p_{B}\left(x_{i}\right), v_{B}\left(x_{i}\right)\right\rangle$ are two single valued neutrosophic sets in the universe of universe $X=\left\{x_{1}, x_{2}, \ldots, x_{n}\right\}$. Then the single valued neutrosophic cross entropy is defined by

$$
\begin{aligned}
& H_{S V N S}(A, B)=\sum_{i=1}^{n}\left[u_{A}\left(x_{i}\right) \log _{2} \frac{u_{A}\left(x_{i}\right)}{\frac{1}{2}\left(u_{A}\left(x_{i}\right)+u_{B}\left(x_{i}\right)\right)}+\left(1-u_{A}\left(x_{i}\right)\right) \log _{2} \frac{1-u_{A}\left(x_{i}\right)}{1-\frac{1}{2}\left(u_{A}\left(x_{i}\right)+u_{B}\left(x_{i}\right)\right)}\right] \\
& \quad+\left[p_{A}\left(x_{i}\right) \log _{2} \frac{p_{A}\left(x_{i}\right)}{\frac{1}{2}\left(p_{A}\left(x_{i}\right)+p_{B}\left(x_{i}\right)\right)}+\left(1-p_{A}\left(x_{i}\right)\right) \log _{2} \frac{1-p_{A}\left(x_{i}\right)}{1-\frac{1}{2}\left(p_{A}\left(x_{i}\right)+p_{B}\left(x_{i}\right)\right)}\right] \\
& \quad+\left[v_{A}\left(x_{i}\right) \log _{2} \frac{v_{A}\left(x_{i}\right)}{\frac{1}{2}\left(v_{A}\left(x_{i}\right)+v_{B}\left(x_{i}\right)\right)}+\left(1-v_{A}\left(x_{i}\right)\right) \log _{2} \frac{1-v_{A}\left(x_{i}\right)}{1-\frac{1}{2}\left(v_{A}\left(x_{i}\right)+v_{B}\left(x_{i}\right)\right)}\right]
\end{aligned}
$$

which measures the degree of discrimination of $A$ from $B$. By considering Shannon's inequality ([16]), one can easily show that $H_{S V N S}(A, B) \geq 0$ and $H_{S V N S}(A, B)=0$ iff $u_{A}\left(x_{i}\right)=u_{B}\left(x_{i}\right), p_{A}\left(x_{i}\right)=p_{B}\left(x_{i}\right)$ and $v_{A}\left(x_{i}\right)=v_{B}\left(x_{i}\right)$ for $x_{i} \in X$. Additionally, $H_{S V N S}(A, B)$ doesn't have symmetry property. So it should be transformed to a symmetric discrimination information measure for single valued neutrosophic sets as $I_{S V N S}(A, B)=H_{S V N S}(A, B)+H_{S V N S}(B, A)$. Thus, if $I_{S V N S}(A, B)$ is the larger, then the difference between $A$ and $B$ is the larger.

\subsection{Interval neutrosophic cross entropy based on fuzzy cross-entropy}


In the section, we propose a method to convert any interval neutrosophic set into a fuzzy set

Definition 3.5 Let $A$ be an interval neutrosophic set in universe $X=\left\{x_{1}, x_{2}, \ldots, x_{n}\right\}$, where

$$
A=\left\langle\left[u_{A}^{L}(x), u_{A}^{U}(x)\right],\left[p_{A}^{L}(x), p_{A}^{U}(x)\right],\left[v_{A}^{L}(x), v_{A}^{U}(x)\right]\right\rangle
$$

Then the average possible membership degree of element $x$ to interval neutrosophic set $A$ can be defined as

$$
\begin{aligned}
\tilde{\mu}_{A}(x) & =\frac{1}{3}\left[\frac{u_{A}^{L}(x)+u_{A}^{U}(x)}{2}+1-\frac{p_{A}^{L}(x)+p_{A}^{U}(x)}{2}+1-\frac{v_{A}^{L}(x)+v_{A}^{U}(x)}{2}\right] \\
& =\frac{u_{A}^{L}(x)+u_{A}^{U}(x)+4-p_{A}^{L}(x)-p_{A}^{U}(x)-v_{A}^{L}(x)-v_{A}^{U}(x)}{6} .
\end{aligned}
$$

Obviously, $F=\left\{\left\langle x, \tilde{\mu}_{A}(x)\right\rangle: x \in X\right\}$ is a fuzzy set in universe $X$.

Example 3.6 Assume that $X=\left\{x_{1}, x_{2}\right\}$ is a universe set including only two elements and $A=$ $\left\langle\left(x_{1},[0.6,0.7],[0.1,0.2],[0.2,0.3]\right),\left(x_{2},[0.4,0.5],[0.2,0.4],[0.7,0.9]\right): x_{1}, x_{2} \in X\right\rangle$.

Then $F=\left\{\left\langle\left(x_{1}, 0.75\right),\left(x_{2}, 0.45\right)\right\rangle: x \in X\right\}$ is a fuzzy set in universe $X$ corresponding to interval neutrosophic set $A$.

Similar to the cross entropy of fuzzy sets, we give the definition of the cross-entropy between two interval neutrosophic sets $A$ and $B$ as follows.

Definition 3.7 Let $A$ and $B$ be two interval neutrosophic sets in universe $X$ and $x \in X$, such that

$$
\begin{aligned}
& A=\left\langle\left[u_{A}^{L}(x), u_{A}^{U}(x)\right],\left[p_{A}^{L}(x), p_{A}^{U}(x)\right],\left[v_{A}^{L}(x), v_{A}^{U}(x)\right]\right\rangle \\
& B=\left\langle\left[u_{B}^{L}(x), u_{B}^{U}(x)\right],\left[p_{B}^{L}(x), p_{B}^{U}(x)\right],\left[v_{B}^{L}(x), v_{B}^{U}(x)\right]\right\rangle
\end{aligned}
$$

Then the average possible membership degree of element $x$ to interval neutrosophic sets $A$ and $B$ can be, respectively, defined as;

$$
\begin{aligned}
\tilde{\mu}_{A}(x) & =\frac{1}{3}\left[\frac{u_{A}^{L}(x)+u_{A}^{U}(x)}{2}+1-\frac{p_{A}^{L}(x)+p_{A}^{U}(x)}{2}+1-\frac{v_{A}^{L}(x)+v_{A}^{U}(x)}{2}\right] \\
& =\frac{u_{A}^{L}(x)+u_{A}^{U}(x)+4-p_{A}^{L}(x)-p_{A}^{U}(x)-v_{A}^{L}(x)-v_{A}^{U}(x)}{6} \\
\tilde{\mu}_{B}(x) & =\frac{1}{3}\left[\frac{u_{B}^{L}(x)+u_{B}^{U}(x)}{2}+1-\frac{p_{B}^{L}(x)+p_{B}^{U}(x)}{2}+1-\frac{v_{B}^{L}(x)+v_{B}^{U}(x)}{2}\right] \\
& =\frac{u_{B}^{L}(x)+u_{B}^{U}(x)+4-p_{B}^{L}(x)-p_{B}^{U}(x)-v_{B}^{L}(x)-v_{B}^{U}(x)}{6} .
\end{aligned}
$$

So, the interval neutrosophic cross-entropy based on fuzzy cross-entropy is defined by

$$
\begin{gathered}
H_{I N S}(A, B)=\sum_{i=1}^{n}\left(\left(\frac{u_{A}^{L_{i}}+u_{A}^{U_{i}}+4-p_{A}^{L_{i}}-p_{A}^{U_{i}}-v_{A}^{L_{i}}-v_{A}^{U_{i}}}{6}\right) \times\right. \\
\log _{2}\left(\frac{2\left(u_{A}^{L_{i}}+u_{A}^{U_{i}}+4-p_{A}^{L_{i}}-p_{A}^{U_{i}}-v_{A}^{L_{i}}-v_{A}^{U_{i}}\right)}{u_{A}^{L_{i}}+u_{A}^{U_{i}}+4-p_{A}^{L_{i}}-p_{A}^{U_{i}}-v_{A}^{L_{i}}-v_{A}^{U_{i}}+u_{B}^{L_{i}}+u_{B}^{U_{i}}+4-p_{B}^{L_{i}}-p_{B}^{U_{i}}-v_{B}^{L_{i}}-v_{B}^{U_{i}}}\right)+ \\
\left(\frac{p_{A}^{L_{i}}+p_{A}^{U_{i}}+v_{A}^{L_{i}}+v_{A}^{U_{i}}+2-u_{A}^{L_{i}}-u_{A}^{U_{i}}}{6}\right) \times \\
\left.\log _{2}\left(\frac{2\left(p_{A}^{L_{i}}+p_{A}^{U_{i}}+v_{A}^{L_{i}}+v_{A}^{U_{i}}+2-u_{A}^{L_{i}}-u_{A}^{U_{i}}\right)}{p_{A}^{L_{i}}+p_{A}^{U_{i}}+v_{A}^{L_{i}}+v_{A}^{U_{i}}+2-u_{A}^{L_{i}}-u_{A}^{U_{i}}+p_{B}^{L_{i}}+p_{B}^{U_{i}}+v_{B}^{L_{i}}+v_{B}^{U_{i}}+2-u_{B}^{L_{i}}-u_{B}^{U_{i}}}\right)\right)
\end{gathered}
$$


which indicates discrimination degree of $A$ from $B$. Here $u_{A}^{L_{i}}$ denotes the $u_{A}^{L}\left(x_{i}\right)$, the same as other notions such as $u_{A}^{U_{i}}, p_{A}^{L_{i}}, p_{A}^{U_{i}}, v_{A}^{L_{i}}, v_{A}^{U_{i}}, u_{B}^{L_{i}}, u_{B}^{U_{i}}, p_{B}^{L_{i}}, p_{B}^{U_{i}}, v_{B}^{L_{i}}, v_{B}^{U_{i}}$.

Then we simply can give the cross-entropy as

$$
H_{I N S}(A, B)=\sum_{i=1}^{n}\left(\tilde{\mu}_{A}\left(x_{i}\right) \log _{2} \frac{\tilde{\mu}_{A}\left(x_{i}\right)}{\frac{1}{2}\left(\tilde{\mu}_{A}\left(x_{i}\right)+\tilde{\mu}_{B}\left(x_{i}\right)\right)}+\left(1-\tilde{\mu}_{A}\left(x_{i}\right)\right) \log _{2} \frac{1-\tilde{\mu}_{A}\left(x_{i}\right)}{1-\frac{1}{2}\left(\tilde{\mu}_{A}\left(x_{i}\right)+\tilde{\mu}_{B}\left(x_{i}\right)\right)}\right),
$$

which $H_{I N S}(A, B)$ indicates the degree of discrimination of $A$ from $B$.

However, it doesn't have symmetry property. So, it should be transformed to a symmetric form of cross entropy for interval neutrosophic sets as follows: $I_{I N S}(A, B)=H_{I N S}(A, B)+H_{I N S}(B, A)$.

Based on Shannon's inequality ([16]), one can easily show that $H_{I N S}(A, B) \geq 0$ and $H_{I N S}(A, B)=0$ iff $u_{A}(x)=p_{A}(x)=v_{A}(x) \quad$ that $\quad$ is, $\quad\left[u_{A}^{L}(x), u_{A}^{U}(x)\right]=\left[u_{B}^{L}(x), u_{B}^{U}(x)\right],\left[p_{A}^{L}(x), p_{A}^{U}(x)\right]=\left[p_{B}^{L}(x), p_{B}^{U}(x)\right]$ and $\left[v_{A}^{L}(x), v_{A}^{U}(x)\right]=\left[v_{B}^{L}(x), v_{B}^{U}(x)\right]$, for $x_{i} \in X$. Moreover, it is clear that $I_{I N S}(A, B)=I_{I N S}\left(A^{c}, B^{c}\right)$ and $I_{I N S}(A, B)=I_{I N S}(B, A)$.

On the other hand, the weighted cross-entropy measure between an alternative $A_{i}$ and the ideal alternative $A^{*}$ can be expressed as

$$
H_{I N S \omega}\left(A^{*}, A_{i}\right)=\sum_{j=1}^{n} \omega_{j}\left(\tilde{\mu}_{A^{*}}\left(x_{j}\right) \log _{2} \frac{\tilde{\mu}_{A^{*}}\left(x_{j}\right)}{\frac{1}{2}\left(\tilde{\mu}_{A^{*}}\left(x_{j}\right)+\tilde{\mu}_{A_{i}}\left(x_{j}\right)\right)}+\left(1-\tilde{\mu}_{A^{*}}\left(x_{j}\right)\right) \log _{2} \frac{1-\tilde{\mu}_{A^{*}}\left(x_{j}\right)}{1-\frac{1}{2}\left(\tilde{\mu}_{A^{*}}\left(x_{j}\right)+\tilde{\mu}_{A_{i}}\left(x_{j}\right)\right)}\right),
$$

whose weight vector is $\omega=\left(\omega_{1}, \omega_{2}, \ldots, \omega_{n}\right)^{T}$ such that $\omega_{j} \in[0,1], \sum_{j=1}^{n} \omega_{j}=1$.

It is easily proved that $I_{I N S \omega}(A, B)=H_{I N S \omega}(A, B)+H_{I N S \omega}(B, A)$ for the interval neutrosophic sets $A$ and $B$.

\subsection{Interval neutrosophic cross entropy based on single valued neutrosophic cross-entropy}

In this subsection, we develop another technic to compute the cross entropy of interval valued neutrosophic sets. The approach is based on the reduction of the interval neutrosophic sets. To transform the interval neutrosophic sets into single valued neutrosophic sets, we propose the following reduction operator.

Definition 3.8 Assume that $A=\left\langle\left[u_{A}^{L}(x), u_{A}^{U}(x)\right],\left[p_{A}^{L}(x), p_{A}^{U}(x)\right],\left[v_{A}^{L}(x), v_{A}^{U}(x)\right]\right\rangle$ is an interval neutrosophic set and $f_{\lambda}: \operatorname{INS}(X) \rightarrow \operatorname{SVNS}(X)$ is a mapping given by

$$
f_{\lambda}(A)=\left\langle u_{A}^{L}(x)+\lambda \Delta u_{A}(x), p_{A}^{L}(x)+(1-\lambda) \Delta p_{A}(x), v_{A}^{L}(x)+(1-\lambda) \Delta v_{A}(x)\right\rangle,
$$

where $\Delta u_{A}(x)=u_{A}^{U}(x)-u_{A}^{L}(x), \Delta p_{A}(x)=p_{A}^{U}(x)-p_{A}^{L}(x)$ and $\Delta v_{A}(x)=v_{A}^{U}(x)-v_{A}^{L}(x)$ for $x \in X$ and $\lambda \in[0,1]$. Then $f_{\lambda}$ is called a reduction operator which is used to assign a single valued neutrosophic set to an interval neutrosophic set.

Obviously, $A_{\lambda}$ is a single valued neutrosophic set in universe $X$.

Example 3.9 Assume that $X=\left\{x_{1}\right\}$ is a universe set including only one element and $A=\left\langle\left(x_{1},[0.2,0.5],[0.1,0.3],[0.2,0.6]\right): x_{1} \in X\right\rangle$ and $B=\left\langle\left(x_{1},[0.3,0.5],[0.1,0.4],[0.3,0.4]\right): x_{1} \in X\right\rangle$. For $\lambda=0.5$, we can compute the single valued neutrosophic sets $f_{0.5}(A)$ and $f_{0.5}(B)$ as follows

$$
\begin{aligned}
& f_{0.5}(A)=\left\langle\left(x_{1}, 0.35,0.20,0.40\right): x_{1} \in X\right\rangle \\
& f_{0.5}(B)=\left\langle\left(x_{1}, 0.40,0.25,0.35\right): x_{1} \in X\right\rangle
\end{aligned}
$$

Proposition 3.10 Let $A$ and $B$ two interval neutrosophic sets in universe $X=\left\{x_{1}, x_{2}, \ldots, x_{n}\right\}$, where 


$$
\begin{aligned}
A & =\left\langle\left[u_{A}^{L}(x), u_{A}^{U}(x)\right],\left[p_{A}^{L}(x), p_{A}^{U}(x)\right],\left[v_{A}^{L}(x), v_{A}^{U}(x)\right]\right\rangle, \\
B & =\left\langle\left[u_{B}^{L}(x), u_{B}^{U}(x)\right],\left[p_{B}^{L}(x), p_{B}^{U}(x)\right],\left[v_{B}^{L}(x), v_{B}^{U}(x)\right]\right\rangle
\end{aligned}
$$

Assume $f_{\lambda}: \operatorname{INS}(X) \rightarrow \operatorname{SVNS}(X)$ is a reduction operator and $\lambda, \delta \in[0,1]$. Then

(1) if $0 \leq \lambda \leq \delta$, then $f_{\lambda}(A) \subseteq f_{\delta}(A)$;

(2) if $A \subseteq B$, then $f_{\lambda}(A) \subseteq f_{\lambda}(B)$;

(3) $f_{\lambda}\left(f_{\delta}(A)\right)=f_{\delta}(A)$;

(4) $\left(f_{\lambda}\left(A^{c}\right)\right)^{c}=f_{1-\lambda}(A)$.

\section{Proof:}

(1) Assume that $0 \leq \lambda \leq \delta$ for $\lambda, \delta \in[0,1]$. Then we have

$$
\begin{aligned}
& f_{\lambda}(A)=\left\langle u_{A}^{L}(x)+\lambda \Delta u_{A}(x), p_{A}^{L}(x)+(1-\lambda) \Delta p_{A}(x), v_{A}^{L}(x)+(1-\lambda) \Delta v_{A}(x)\right\rangle, \\
& f_{\delta}(A)=\left\langle u_{A}^{L}(x)+\delta \Delta u_{A}(x), p_{A}^{L}(x)+(1-\delta) \Delta p_{A}(x), v_{A}^{L}(x)+(1-\delta) \Delta v_{A}(x)\right\rangle,
\end{aligned}
$$

where,

$$
\begin{gathered}
u_{A}^{L}(x)+\lambda \Delta u_{A}(x) \leq u_{A}^{L}(x)+\delta \Delta u_{A}(x), \\
p_{A}^{L}(x)+(1-\delta) \Delta p_{A}(x) \leq p_{A}^{L}(x)+(1-\lambda) \Delta p_{A}(x), \\
v_{A}^{L}(x)+(1-\delta) \Delta v_{A}(x) \leq v_{A}^{L}(x)+(1-\lambda) \Delta v_{A}(x) .
\end{gathered}
$$

So, $f_{\lambda}(A) \subseteq f_{\delta}(A)$.

(2) Assume that $A \subseteq B$. Then we have $u_{A}^{L}(x) \leq u_{B}^{L}(x), u_{A}^{U}(x) \leq u_{B}^{U}(x), p_{A}^{L}(x) \geq p_{B}^{L}(x), p_{A}^{U}(x) \geq p_{B}^{U}(x)$ and $v_{A}^{L}(x) \geq v_{B}^{L}(x), v_{A}^{U}(x) \geq v_{B}^{U}(x)$ for all $x \in X$. It implies that

$$
\begin{gathered}
u_{A}^{L}(x)+\lambda \Delta u_{A}(x) \leq u_{B}^{L}(x)+\lambda \Delta u_{B}(x), \\
p_{B}^{L}(x)+(1-\lambda) \Delta p_{B}(x) \leq p_{A}^{L}(x)+(1-\lambda) \Delta p_{A}(x), \\
v_{B}^{L}(x)+(1-\lambda) \Delta v_{B}(x) \leq v_{A}^{L}(x)+(1-\lambda) \Delta v_{A}(x) .
\end{gathered}
$$

So, $f_{\lambda}(A) \subseteq f_{\lambda}(B)$.

(3) It is easily proved that $f_{\lambda}\left(f_{\delta}(A)\right)=f_{\delta}(A)$.

(4) Let $A \in I N S(X)$ and so $A^{c}=\left\langle\left[v_{A}^{L}(x), v_{A}^{U}(x)\right],\left[1-p_{A}^{U}(x), 1-p_{A}^{L}(x)\right],\left[u_{A}^{L}(x), u_{A}^{U}(x)\right]\right\rangle$ for each $x \in X$. Then we have the following

$$
f_{\lambda}\left(A^{c}\right)=\left\langle v_{A}^{L}(x)+\lambda \Delta v_{A}(x), 1-p_{A}^{U}(x)+(1-\lambda) \Delta p_{A}(x), u_{A}^{L}(x)+(1-\lambda) \Delta u_{A}(x)\right\rangle
$$

for each $x \in X$. Hence, it follows that

$$
\begin{aligned}
\left(f_{\lambda}\left(A^{c}\right)\right)^{c}= & \left\langle u_{A}^{L}(x)+(1-\lambda) \Delta u_{A}(x), 1-\left(1-p_{A}^{U}(x)+(1-\lambda) \Delta p_{A}(x)\right), v_{A}^{L}(x)+\lambda \Delta v_{A}(x)\right\rangle \\
= & \left\langle u_{A}^{L}(x)+(1-\lambda) \Delta u_{A}(x), 1-\left(1-p_{A}^{U}(x)+\left(p_{A}^{U}(x)-p_{A}^{L}(x)\right)-\lambda \Delta p_{A}(x)\right), v_{A}^{L}(x)\right. \\
& \left.\quad+\lambda \Delta v_{A}(x)\right\rangle \\
= & \left\langle u_{A}^{L}(x)+(1-\lambda) \Delta u_{A}(x), p_{A}^{L}(x)+\lambda \Delta p_{A}(x), v_{A}^{L}(x)+\lambda \Delta v_{A}(x)\right\rangle \\
= & f_{1-\lambda}(A) .
\end{aligned}
$$

Similar to the cross entropy of single valued neutrosophic sets, we define the concept of the cross entropy based on the reduction of the interval neutrosophic sets, which is a generalization of single valued neutrosophic cross entropy, as follows:

Definition 3.11 Let $A, B \in I N S(X)$ and $X=\left\{x_{1}, x_{2}, \ldots, x_{n}\right\}$. Then interval neutrosophic cross entropy based on single valued neutrosophic cross entropy is defined by

$$
E_{I N S}(A, B)=\sum_{i=1}^{n}\left[\bar{u}_{A}\left(x_{i}\right) \log _{2} \frac{\bar{u}_{A}\left(x_{i}\right)}{\frac{1}{2}\left(\bar{u}_{A}\left(x_{i}\right)+\bar{u}_{B}\left(x_{i}\right)\right)}+\left(1-\bar{u}_{A}\left(x_{i}\right)\right) \log _{2} \frac{1-\bar{u}_{A}\left(x_{i}\right)}{1-\frac{1}{2}\left(\bar{u}_{A}\left(x_{i}\right)+\bar{u}_{B}\left(x_{i}\right)\right)}\right]
$$




$$
\begin{aligned}
& +\left[\bar{p}_{A}\left(x_{i}\right) \log _{2} \frac{\bar{p}_{A}\left(x_{i}\right)}{\frac{1}{2}\left(\bar{p}_{A}\left(x_{i}\right)+\bar{p}_{B}\left(x_{i}\right)\right)}+\left(1-\bar{p}_{A}\left(x_{i}\right)\right) \log _{2} \frac{1-\bar{p}_{A}\left(x_{i}\right)}{1-\frac{1}{2}\left(\bar{p}_{A}\left(x_{i}\right)+\bar{p}_{B}\left(x_{i}\right)\right)}\right] \\
& +\left[\bar{v}_{A}\left(x_{i}\right) \log _{2} \frac{\bar{v}_{A}\left(x_{i}\right)}{\frac{1}{2}\left(\bar{v}_{A}\left(x_{i}\right)+\bar{v}_{B}\left(x_{i}\right)\right)}+\left(1-\bar{v}_{A}\left(x_{i}\right)\right) \log _{2} \frac{1-\bar{v}_{A}\left(x_{i}\right)}{1-\frac{1}{2}\left(\bar{v}_{A}\left(x_{i}\right)+\bar{v}_{A}\left(x_{i}\right)\right)}\right]
\end{aligned}
$$

where $\bar{u}_{A}\left(x_{i}\right)=u_{A}^{L}(x)+\lambda \Delta u_{A}(x), \bar{p}_{A}\left(x_{i}\right)=p_{A}^{L}(x)+\lambda \Delta p_{A}(x)$ and $\bar{v}_{A}\left(x_{i}\right)=v_{A}^{L}(x)+\lambda \Delta v_{A}(x)$, and similarly, $\bar{u}_{B}\left(x_{i}\right)=u_{B}^{L}(x)+\lambda \Delta u_{B}(x), \bar{p}_{B}\left(x_{i}\right)=p_{B}^{L}(x)+\lambda \Delta p_{B}(x)$ and $\bar{v}_{B}\left(x_{i}\right)=v_{B}^{L}(x)+\lambda \Delta v_{B}(x)$ for all for $x \in X$ and $\lambda \in[0,1]$, which $\lambda$ is the threshold value.

Unless otherwise stated, we choose the center value, i.e., $\lambda=0,5$.

Based on Shannon's inequality ([16]), one can easily show that $E_{I N S}(A, B) \geq 0$ and $E_{I N S}(A, B)=0$ iff $u_{A}(x)=p_{A}(x)=v_{A}(x) \quad$ that $\quad$ is, $\quad\left[u_{A}^{L}(x), u_{A}^{U}(x)\right]=\left[u_{B}^{L}(x), u_{B}^{U}(x)\right],\left[p_{A}^{L}(x), p_{A}^{U}(x)\right]=\left[p_{B}^{L}(x), p_{B}^{U}(x)\right]$ $\operatorname{and}\left[v_{A}^{L}(x), v_{A}^{U}(x)\right]=\left[v_{B}^{L}(x), v_{B}^{U}(x)\right]$ for all $x \in X$. Moreover, $E_{I N S}(A, B)$ doesn't have symmetry property. So it should be transformed to a symmetric discrimination information measure for interval neutrosophic sets as $J_{I N S}(A, B)=E_{I N S}(A, B)+E_{I N S}(B, A)$. Thus, if $J_{S V N S}(A, B)$ is the larger, then the difference between $A$ and $B$ is the larger.

On the other hand, the weighted cross-entropy measure between an alternative $A_{i}$ and the ideal alternative $A^{*}$ can be expressed as

$$
\begin{aligned}
E_{I N S \omega}\left(A^{*}, A_{i}\right)=\sum_{j=1}^{n} \omega_{j}\left[\bar{u}_{A^{*}}\left(x_{j}\right) \log _{2} \frac{\bar{u}_{A^{*}}\left(x_{j}\right)}{\frac{1}{2}\left(\bar{u}_{A^{*}}\left(x_{j}\right)+\bar{u}_{A_{i}}\left(x_{i}\right)\right)}+\left(1-\bar{u}_{A^{*}}\left(x_{j}\right)\right) \log _{2} \frac{1-\bar{u}_{A^{*}}\left(x_{j}\right)}{1-\frac{1}{2}\left(\bar{u}_{A^{*}}\left(x_{j}\right)+\bar{u}_{A_{i}}\left(x_{j}\right)\right)}\right] \\
+\left[\bar{p}_{A^{*}}\left(x_{j}\right) \log _{2} \frac{\bar{p}_{A^{*}}\left(x_{j}\right)}{\frac{1}{2}\left(\bar{p}_{A^{*}}\left(x_{j}\right)+\bar{p}_{A_{i}}\left(x_{j}\right)\right)}+\left(1-\bar{p}_{A^{*}}\left(x_{j}\right)\right) \log _{2} \frac{1-\bar{p}_{A^{*}}\left(x_{j}\right)}{1-\frac{1}{2}\left(\bar{p}_{A^{*}}\left(x_{j}\right)+\bar{p}_{A_{i}}\left(x_{j}\right)\right)}\right] \\
+\left[\bar{v}_{A^{*}}\left(x_{i}\right) \log _{2} \frac{\bar{v}_{A^{*}}\left(x_{j}\right)}{\frac{1}{2}\left(\bar{v}_{A^{*}}\left(x_{j}\right)+\bar{v}_{A_{i}}\left(x_{j}\right)\right)}+\left(1-\bar{v}_{A^{*}}\left(x_{j}\right)\right) \log _{2} \frac{1-\bar{v}_{A^{*}}\left(x_{j}\right)}{1-\frac{1}{2}\left(\bar{v}_{A^{*}}\left(x_{j}\right)+\bar{v}_{A_{i}}\left(x_{j}\right)\right)}\right],(10)
\end{aligned}
$$

whose weight vector is $\omega=\left(\omega_{1}, \omega_{2}, \ldots, \omega_{n}\right)^{T}$ such that $\omega_{j} \in[0,1], \sum_{j=1}^{n} \omega_{j}=1$.

It is easily proved that $J_{I N S \omega}(A, B)=E_{I N S \omega}(A, B)+E_{I N S \omega}(B, A)$ for the interval neutrosophic sets $A$ and $B$.

\section{Multi-criteria neutrosophic decision-making method based on the cross entropy}

As a new branch of neutrosophic sets, interval neutrosophic set can be used to solve problems including uncertain, imprecise, inconsistent and incomplete information existing in real scientific, environment and engineering applications. It has drawn the attention of many researchers for handling uncertainty. In this section, an illustrative example adopted by ([32]) is given to verify the proposed approach and to demonstrate its practicality and effectiveness.

Let $A=\left\{A_{1}, A_{2}, \ldots, A_{m}\right\}$ be the set of alternatives and $C=\left\{C_{1}, C_{2}, \ldots, C_{n}\right\}$ the set of attributes, whose weight vector is $\omega=\left(\omega_{1}, \omega_{2}, \ldots, \omega_{n}\right)^{T}$ such that $\omega_{j} \in[0,1], \sum_{j=1}^{n} \omega_{j}=1$. Thus, the characteristic of the alternative $A_{i}(i=1,2, \ldots, m)$ is expressed by an interval neutrosophic set:

$$
A_{i}=\left\{\left\langle x,\left[u_{A_{i}}^{L}\left(C_{j}\right), u_{A_{i}}^{U}\left(C_{j}\right)\right],\left[p_{A_{i}}^{L}\left(C_{j}\right), p_{A_{i}}^{U}\left(C_{j}\right)\right],\left[v_{A_{i}}^{L}\left(C_{j}\right), v_{A_{i}}^{U}\left(C_{j}\right)\right]\right\rangle: C_{j} \in C\right\}
$$


where $0 \leq u_{A_{i}}^{U}\left(C_{j}\right)+p_{A_{i}}^{U}\left(C_{j}\right)+v_{A_{i}}^{U}\left(C_{j}\right) \leq 3, u_{A_{i}}^{L}\left(C_{j}\right) \geq 0, p_{A_{i}}^{U}\left(C_{j}\right) \geq 0, \quad v_{A_{i}}^{L}\left(C_{j}\right) \geq 0, j=1,2, \ldots, n$ and $i=1,2, \ldots, n$. Here, $\left[u_{A_{i}}^{L}\left(C_{j}\right), u_{A_{i}}^{U}\left(C_{j}\right)\right]$ indicates the degree that the alternative $A_{i}$ satisfies the criterion $C_{j}$ and $\left[p_{A_{i}}^{L}\left(C_{j}\right), p_{A_{i}}^{U}\left(C_{j}\right)\right]$ indicates the degree that the alternative $A_{i}$ is indeterminacy on the criterion $C_{j}$, where as $\left[v_{A_{i}}^{L}\left(C_{j}\right), v_{A_{i}}^{U}\left(C_{j}\right)\right]$ indicates the degree that the alternative $A_{i}$ does not satisfy the criterion $C_{j}$ given by the decision-maker. So we can express a decision matrix $A=\left(\alpha_{i j}\right)_{m \times n}$. Additionally, in multicriteria decision making process, the concept of ideal point has been used to help determine the best alternative in the decision set. Although the ideal alternative that does not exist in real life, it does provide a useful theoretical construct against which to evaluate alternatives. Here, we can define an ideal fuzzy value and an ideal single valued neutrosophic value, respectively as $A^{*}=\langle(x, 1)\rangle$ and $A^{*}=\langle(x, 1,0,0)\rangle$ for all $x \in X$. Also, the positive-ideal solution $A^{+}$for single valued neutrosophic sets is a single valued neutrosophic value defined by

$$
A^{+}=\left\langle\left(u_{A}^{+}\left(C_{j}\right), p_{A}^{+}\left(C_{j}\right), v_{A}^{+}\left(C_{j}\right)\right)\right\rangle
$$

where $u_{A^{+}}\left(C_{j}\right)=\max _{i} u_{A_{i}}\left(C_{j}\right), p_{A^{+}}\left(C_{j}\right)=\min _{i} p_{A_{i}}\left(C_{j}\right)$ and $v_{A^{+}}\left(C_{j}\right)=\min _{i} v_{A_{i}}\left(C_{j}\right)$, for $i=1,2, \ldots, m$ and $j=1,2, \ldots, n$. It is clear that the positive-ideal solution $A^{+}$for fuzzy sets is a fuzzy value defined by $A^{+}=$ $\left\langle\left(\mu_{A}^{+}\left(C_{j}\right)\right)\right\rangle$, where $\mu_{A^{+}}\left(C_{j}\right)=\max _{i} \mu_{A_{i}}\left(C_{j}\right)$.

Therefore, the smaller the value of $\mathrm{I}_{\mathrm{INS}}\left(A^{*}, A_{i}\right)$ (or $\left.\mathrm{JINS}_{\mathrm{INS}}\left(A^{*}, A_{i}\right)\right)$ is, the better the alternative $A_{i}$ is. In this case, the alternative $A_{i}$ is closer to the ideal alternative $A^{*}$. Through the weighted cross entropy $\operatorname{INS} \omega_{\mathrm{IN}}\left(A^{*}, A_{i}\right)$ (or $\left.\mathrm{J}_{\mathrm{INS} \omega}\left(A^{*}, A_{i}\right)\right)(i=1,2, \ldots, m)$ between each alternative and the ideal alternative, we can determine the ranking order of all alternatives and can easily select the best one. Moreover, we mean similar thoughts for the positive-ideal solution $A^{+}$.

In the following, we can summary the decision procedure for two developed methods to compute the cross-entropy of interval neutrosophic sets, which are called the interval neutrosophic cross entropy based on extension of single valued neutrosophic cross entropy and fuzzy cross-entropy, respectively.

Method (1) Interval neutrosophic cross entropy based on fuzzy cross-entropy

Step 1. Obtain the fuzzy sets with the average possible membership corresponding to interval neutrosophic sets by using Eq. (4).

Step 2. Calculate the symmetric form of cross entropy $I_{I N S \omega}\left(A^{*}, A_{i}\right)\left(\right.$ or $\left.I_{I N S \omega}\left(A^{+}, A_{i}\right)\right)$ for interval neutrosophic set $A_{i}(i=1,2, \ldots, m)$ by using Eq. (7).

Step 3. Rank the alternatives $A_{i}(i=1,2, \ldots, m)$ and select to best one $(s)$ in according to $I_{I N S \omega}\left(A^{*}, A_{i}\right)\left(\right.$ or $\left.I_{I N S \omega}\left(A^{+}, A_{i}\right)\right)$ for $i=1,2, \ldots, m$.

Method (2) Interval neutrosophic cross entropy based on single valued neutrosophic cross-entropy

Step 1. Obtain the single valued neutrosophic sets based on the threshold value corresponding to interval neutrosophic sets by using Eq. (8).

Step 2. Calculate the symmetric form of cross entropy $J_{I N S \omega}\left(A^{*}, A_{i}\right)\left(\right.$ or $\left.J_{I N S \omega}\left(A^{+}, A_{i}\right)\right)$ for singel valued neutrosophic set $A_{i}(i=1,2, \ldots, m)$ by using Eq. (10).

Step 3. Rank the alternatives $A_{i}(i=1,2, \ldots, m)$ and select to best one(s) in according to the symmetric form $J_{I N S \omega}\left(A^{*}, A_{i}\right)\left(\right.$ or $\left._{I N S \omega}\left(A^{+}, A_{i}\right)\right)$ for $i=1,2, \ldots, m$.

\subsection{Numerical example}


Example 4.1 Let us consider decision making problem adapted from ([32]). Suppose that there is a panel with four possible alternatives to invest the money: (1) $A_{1}$ is a food company; (2) $A_{2}$ is a car company; (3) $A_{3}$ is an arms company; (4) $A_{4}$ is a computer company. The investment company must make a decision according to three criteria given below: (1) $C_{1}$ is the growth analysis; (2) $C_{2}$ is the risk analysis; (3) $C_{3}$ is the environmental impact analysis. By using the interval-valued intuitionistic fuzzy information, the decision-maker has evaluated the four possible alternatives under the above three criteria and has listed in the following matrix:

$$
\begin{array}{cccc} 
& C_{1} & C_{2} & C_{3} \\
A_{1} & ([0.4,0.5],[0.2,0.3],[0.3,0.4]) & ([0.4,0.6],[0.1,0.3],[0.2,0.4]) & ([0.7,0.9],[0.2,0.3],[0.4,0.5]) \\
A_{2} & ([0.6,0.7],[0.1,0.2],[0.2,0.3]) & ([0.6,0.7],[0.1,0.2],[0.2,0.3]) & ([0.3,0.6],[0.3,0.5],[0.8,0.9]) \\
A_{3} & ([0.3,0.6],[0.2,0.3],[0.3,0.4]) & ([0.5,0.6],[0.2,0.3],[0.3,0.4]) & ([0.4,0.5],[0.2,0.4],[0.7,0.9]) \\
A_{4} & ([0.7,0.8],[0.0,0.1],[0.1,0.2]) & ([0.6,0.7],[0.1,0.2],[0.1,0.3]) & ([0.6,0.7],[0.3,0.4],[0.8,0.9])
\end{array}
$$

Suppose that the weights of $C_{1}, C_{2}$ and $C_{3}$ are $0.35,0.25$ and 0.40 . Then, we use the approach proposed to obtain the most desirable alternative(s).

Method (1) Interval neutrosophic cross entropy based on fuzzy crosss-entropy:

Step 1. We can obtain the fuzzy sets with the average possible membership corresponding to interval neutrosophic sets by using Eq. (4) as follows:

$$
\begin{aligned}
& A_{1}=\left\{\left\langle\left(C_{1}, 0.6166\right),\left(C_{2}, 0.6666\right),\left(C_{3}, 0.7000\right)\right\rangle\right\}, \\
& A_{2}=\left\{\left\langle\left(C_{1}, 0.7500\right),\left(C_{2}, 0.7500\right),\left(C_{3}, 0.4000\right)\right\rangle\right\}, \\
& A_{3}=\left\{\left\langle\left(C_{1}, 0.6166\right),\left(C_{2} .0,6500\right),\left(C_{3}, 0.4500\right)\right\rangle\right\}, \\
& A_{4}=\left\{\left\langle\left(C_{1}, 0.8500\right),\left(C_{2}, 0.7666\right),\left(C_{3}, 0.4833\right)\right\rangle\right\} .
\end{aligned}
$$

Step 2. By using Eq. (7), we compute $\mathrm{I}_{\operatorname{INS} \omega}\left(\mathrm{A}^{*}, \mathrm{~A}_{i}\right)(\mathrm{i}=1,2, \ldots, \mathrm{m})$ as

$$
\mathrm{I}_{\mathrm{INS} \omega}\left(\mathrm{A}^{*}, \mathrm{~A}_{1}\right)=0.3881, \mathrm{I}_{\mathrm{INS} \omega}\left(\mathrm{A}^{*}, \mathrm{~A}_{2}\right)=0.4822, \mathrm{I}_{\mathrm{INS} \omega}\left(\mathrm{A}^{*}, \mathrm{~A}_{3}\right)=0.5401, \mathrm{I}_{\mathrm{INS} \omega}\left(\mathrm{A}^{*}, \mathrm{~A}_{4}\right)=0.3792 .
$$

Step 3. Rank all alternatives according to the symmetric form $I_{I N S \omega}\left(A^{*}, A_{i}\right)(i=1,2, \ldots, m): A_{4}>A_{1}>$ $\mathrm{A}_{2}>\mathrm{A}_{3}$.

Thus the alternative $A_{4}$ is the most desirable alternative based on the interval neutrosophic cross entropy based on extension, which is also consistent with the result obtain in ([32]).

Now, assuming the same weights for $C_{1}, C_{2}$ and $C_{3}$, we use the interval neutrosophic cross-entropy based on reduction.

Method (2) Interval neutrosophic cross entropy based on single valued neutrosophic cross entropy:

Step (1) We can the single valued neutrosophic sets based on the threshold value corresponding to interval neutrosophic sets by using Eq. (8) as follows:

$$
\begin{aligned}
& A_{1}=\left\{\left\langle\left(C_{1}, 0.45,0.25,0,35\right),\left(C_{2}, 0.50,0.20,0.30\right),\left(C_{3}, 0.80,0.25,0.45\right)\right\rangle\right\}, \\
& A_{2}=\left\{\left\langle\left(C_{1}, 0.65,0.15,0.25\right),\left(C_{2}, 0,65,0.15,0.25\right),\left(C_{3}, 0.45,0.40,0.85\right)\right\rangle\right\}, \\
& A_{3}=\left\{\left\langle\left(C_{1}, 0.45,0.45,0.25\right),\left(C_{2}, 0.55,0.25,0.35\right),\left(C_{3}, 0,45,0.30,0.80\right)\right\rangle\right\}, \\
& A_{4}=\left\{\left\langle\left(C_{1}, 0.75,0.05,0.15\right),\left(C_{2}, 0.65,0.15,0.20\right),\left(C_{3}, 0.65,0,35,0.85\right)\right\rangle\right\} .
\end{aligned}
$$

Step (2) By using Eq. (10), we compute $\mathrm{J}_{\mathrm{INS} \omega}\left(\mathrm{A}^{*}, \mathrm{~A}_{\mathrm{i}}\right)(\mathrm{i}=1,2, \ldots, \mathrm{m})$ as

$$
\mathrm{J}_{\mathrm{INS} \omega}\left(\mathrm{A}^{*}, \mathrm{~A}_{1}\right)=1.2924, \mathrm{~J}_{\mathrm{INS} \omega}\left(\mathrm{A}^{*}, \mathrm{~A}_{2}\right)=1.5861, \mathrm{~J}_{\operatorname{INS} \omega}\left(\mathrm{A}^{*}, \mathrm{~A}_{3}\right)=1.8694, \mathrm{~J}_{\operatorname{INS} \omega}\left(\mathrm{A}^{*}, \mathrm{~A}_{4}\right)=1.1252 .
$$


Step (3) Rank all alternatives according to the symmetric form $\operatorname{I}_{\operatorname{INS} \omega}\left(A^{*}, A_{i}\right)(i=1,2, \ldots, m): A_{4}>$ $A_{1}>A_{2}>A_{3}$.

Thus the alternative $A_{4}$ is also the most desirable alternative based on the interval neutrosophic cross entropy, which is also consistent with the result obtain in Method1 and ([32]).

Remark: Ranking the alternatives in accordance with the positive-ideal solution $A^{+}$in Method1, we can compute as

$$
\mathrm{I}_{\mathrm{INS} \omega}\left(\mathrm{A}^{+}, \mathrm{A}_{1}\right)=0.1005, \mathrm{I}_{\mathrm{INS} \omega}\left(\mathrm{A}^{+}, \mathrm{A}_{2}\right)=0.1564, \mathrm{I}_{\mathrm{INS} \omega}\left(\mathrm{A}^{+}, \mathrm{A}_{3}\right)=0.2020, \mathrm{I}_{\mathrm{INS} \omega}\left(\mathrm{A}^{+}, \mathrm{A}_{4}\right)=0.0817
$$

and so we deduce that the priority ranking of all the four alternatives is the same as $A_{4}>A_{1}>A_{2}>A_{3}$. Similarity, by employing the positive-ideal solution $A^{+}$in Method2, we can obtain as

$$
\mathrm{J}_{\mathrm{INS} \omega}\left(\mathrm{A}^{+}, \mathrm{A}_{1}\right)=0.3128, \mathrm{~J}_{\mathrm{INS} \omega}\left(\mathrm{A}^{+}, \mathrm{A}_{2}\right)=0.4136, \mathrm{~J}_{\mathrm{INS} \omega}\left(\mathrm{A}^{+}, \mathrm{A}_{3}\right)=0.6211, \mathrm{~J}_{\mathrm{INS} \omega}\left(\mathrm{A}^{+}, \mathrm{A}_{4}\right)=0.1860 .
$$

Then we have the same ranking order for $\lambda=0.5 ; A_{4}>A_{1}>A_{2}>A_{3}$ and thus the most desirable alternative is $\mathrm{A}_{4}$.

From the examples, since the proposed neutrosophic decision-making method can handle not only incomplete information but also the inconsistent information and indeterminate information existing in real situations, it is more suitable for real scientific and engineering applications. The technique proposed in this paper presents a generalization of existing decision making methods and provides a new way for decision makers.

\section{Conclusions}

Interval neutrosophic set, as a combining concepts of single valued neutrosophic set and interval fuzzy set, provides the additional capability to deal with uncertainty, inconsistent, incomplete and imprecise information by including a truth-membership interval, an indeterminacy-membership interval and a falsity membership interval. Therefore, it has played a significant role in the uncertain system. In this paper, we first proposed two useful methods which are reduce the interval neutrosophic information to single valued neutrosophic information and fuzzy information, respectively. Based on the single valued neutrosophic cross-entropy and the fuzzy cross-entropy, we define the concept of interval neutrosophic cross-entropy by using the two reduction methods. Then the proposed cross-entropy is established in multi criteria decision making problem, which the alternatives on criteria are characterized by interval neutrosophic sets. Finally, a practical example was presented to illustrate the application of the proposed multi criteria decision making.

Since the interval neutrosophic set is a generalization of the aforementioned sets, the proposed method extends previous approaches because of the consideration of indeterminacy information as well as truth and falsity information in the selection process of the alternatives according to criteria. Therefore it is more effective and more remarkable for handling imprecise, inconsistent and incomplete information in multi criteria decision making. Hence, one can easily apply the method to make the optimal choice and to solve multi criteria decision making problems located in other areas such as pattern recognition, clustering analysis, image process and medical diagnoses. In the future, we will continue working on entropy of neutrosophic sets, and so the single valued neutrosophic sets and the interval neutrosophic sets.

\section{References}

[1] Atanassov K (1986) Intuitionistic fuzzy sets. Fuzzy Sets and Systems, (20) 87-96.

[2] Atanassov K (1994) Operators over interval-valued intuitionistic fuzzy sets. Fuzzy Sets and Systems, 64(2) $159-174$.

[3] Atanassov K and Gargov G (1989) Interval-valued intuitionistic fuzzy sets. Fuzzy Sets and Systems, 31(3) 343-349.

[4] Broumi S and Smarandache F (2013) Correlation coefficient of interval neutrosophic set, Appl. Mech. Mater. $436511-517$.

[5] Burillo P and Bustince H (1996) Entropy on intuitionistic fuzzy sets and on interval-valued fuzzy sets, Fuzzy Sets and Systems 78 305-316.

[6] Bhandari D and Pal NR (1993) Some new information measures for fuzzy sets, Information Sciences 67 209-228. 
[7] Cheng HD and Guo Y (2008) A new neutrosophic approach to image thresholding, New Math. Natural Comput. 4 (3) 291308.

[8] DeLuca A and Termini S (1972) A definition of nonprobabilistic entropy in the setting of fuzzy sets theory, Information and Control 20 301-312.

[9] Guo Y and Cheng HD (2009) New neutrosophic approach to image segmentation, Pattern Recognit. 42 587-595.

[10] Liu P and Wang Y (2014) Multiple attribute decision-making method based on single-valued neutrosophic normalized weighted Bonferroni mean, Neural Computing and Applications, 25 (7-8), 2001-2010.

[11] Liu P Shi L (2015) The generalized hybrid weighted average operator based on interval neutrosophic hesitant set and its application to multiple attribute decision making, Neural Computing and Applications, 26 (2) 457-471.

[12] Majumdar P and Samanta SK (2014) On similarity and entropy of neutrosophic sets, J. Intell. Fuzzy Syst. 26 (3) $1245-1252$.

[13] Peng JJ Wang JQ Wang J Zhang HY and Chen XH (2015) Simplified neutrosophic sets and their applications in multi-criteria group decision-making problems, Int. J. Syst. Sci. DOI:10.1080/00207721.2014.994050.

[14] Peng JJ Wang JQ Wu XH Wang J Chen XH (2015) Multi-valued neutrosophic sets and power aggregation operators with their applications in multi-criteria group decision-making problems. International Journal of Computational Intelligence Systems $8(2): 345-363$.

[15] Shang XG and Jiang WS (1997) A note on fuzzy information measures, Pattern Recognit. Lett. 18 425-432.

[16] Shannon CE (1948) A mathematical theory of communication, Bell Syst. Tech. J. 27 379-423.

[17] Şahin R and Küçük A (2014) Subsethood measure for single valued neutrosophic sets, Journal of Intelligent and Fuzzy Systems, DOI: $10.3233 /$ IFS-141304.

[18] Smarandache F (1999) A unifying field in logics. Neutrosophy: Neutrosophic probability, set and logic, American Research Press, Rehoboth.

[19] Smarandache F (2005) A generalization of the intuitionistic fuzzy set. International journal of Pure and Applied Mathematics, 24, 287-297.

[20] Szmidt E and Kacprzyk J (2001) Entropy for intuitionistic fuzzy sets, Fuzzy Sets and Systems 118 467-477.

[21] Turksen, B. (1986). Interval valued fuzzy sets based on normal forms. Fuzzy Sets and Systems, 20, 191-210.

[22] Vlachos IK and Sergiadis GD (2007) Intuitionistic fuzzy information-applications to pattern recognition, Pattern Recognition Letters 28 197-206.

[23] Wei CP, Wang P and Zhang YZ (2011) Entropy, similarity measure of interval-valued intuitionistic fuzzy sets and their applications Information Sciences $1814273-4286$.

[24] Wang H Smarandache F Zhang YQ and Sunderraman R (2010) Single valued neutrosophic sets, Multispace and Multistructure (4) $410-413$.

[25] Wang H Smarandache F Zhang YQ Sunderraman R (2005) Interval neutrosophic sets and logic: theory and applications in computing. Hexis, Arizona.

[26] Ye J (2013) Multicriteria decision-making method using the correlation coefficient under single-valued neutrosophic environment, International Journal of General Systems 42(4) 386-394.

[27] Ye J (2014) Similarity measures between interval neutrosophic sets and their applications in Multi-criteria decision-making. Journal of Intelligent and Fuzzy Systems, 26 165-172.

[28] Ye J (2014) Single valued neutrosophic cross-entropy for multi-criteria decision making problems, Appl. Math. Model. 38 (3) 1170-1175.

[29] Ye J (2014) A multicriteria decision-making method using aggregation operators for simplified neutrosophic sets, J. Intell. Fuzzy Syst. 26 (5) 2459-2466.

[30] Zadeh LA (1965) Fuzzy sets, Inf. Control 8 338-353.

[31] Zadeh LA (1968) Probability measures of fuzzy events, Journal of Mathematical Analysis and Applications 23 $421-427$.

[32] Zhang HY, Wang JQ, Chen XH (2014) Interval neutrosophic sets and their application in multicriteria decision making problems. The Scientific World Journal 2014:645953.

[33] Zhang QS Jiang S Jia B Luo S (2010) Some information measures for interval-valued intuitionistic fuzzy sets, Information Sciences 180 5130-5145. 